\title{
Endoscopic Assisted Coblation Tongue Base Reduction in Patients with Obstructive Sleep Apnea
}

\author{
Yahia Ali abushab ${ }^{1}$, Hani Mohamed Elshami ${ }^{1}$, Abdulghani Eltuhami Albashir ${ }^{2 *}$, Mohamed Hassan Eesa \\ ${ }^{1}$ Department of Otorhinolaryngology, Faculty of Medicine, Zagazig University, Egypt. \\ ${ }^{2}$ Department of Otorhinolaryngology, Faculty of Medicine, Zawia University, Libya \\ *Corresponding Author: Abdulghani Eltuhami Albashir, Tel.: (+218)915451086, Email: abdutuhami83@ gmail.com
}

\begin{abstract}
Background: Obstructive Sleep Apnea (OSA) is the most common sleep breathing disorder. In OSA, breathing is interrupted by a blockage of airflow.

Objective: This study was aimed to assess the updated technique for endoscope-guided coblator tongue base reduction for the treatment of patients with OSA.

Patients and Methods: This clinical trial study included a total of 12 patients with moderate and severe OSA, attending at the Department of Otorhinolaryngology, Faculty of Medicine, Zagazig University Hospital. This study was conducted between April 2018 till August 2019. All the studied patients underwent coblation reduction technique for the treatment of retroglossal obstruction in combination with other palatal and/or nasal surgeries in a multilevel protocol. The tongue base was observed with a 30- degree angled, rigid endoscope (4 mm).

Results: Male gender represents $75 \%$ of the studied patients. Age of the studied patient ranged from 38 to 54 years old with mean age 44.83 years $( \pm 6.41$ ), Oxygen desaturation index (preop.) was ranged from 30.3 to 56.9 , while ODI (postop.) was ranged from 3.8 to 60.9. Conclusions: It could be concluded that tongue base reduction by coblation guided with endoscopy could be safe in management of obstructive sleep apnea without any dangerous complications. Keywords: Obstructive sleep apnea, Coblation, endoscope-guided, Tongue Base Reduction.
\end{abstract}

\section{INTRODUCTION}

Sleep apnea is a disorder characterized by pauses in breathing or periods of shallow breathing during sleep. Each pause can last for a few seconds and they happen many times a night ${ }^{(\mathbf{1})}$. As it disrupts normal sleep, patients affected are often sleepy or tired during the day ${ }^{(2)}$.

Obstructive sleep apnea (OSA) is the most common sleep breathing disorder. In OSA, breathing is interrupted by a blockage of airflow. People with sleep apnea are often not aware they have it. Often it is first observed by a family member ${ }^{(3)}$. Sleep apnea is often diagnosed with an overnight sleep study. For a diagnosis of sleep apnea, more than five episodes per hour must occur ${ }^{(4,5)}$.

OSA results from multilevel collapse of the upper airway, with the tongue base being a common site of obstruction. Multilevel obstructions, including both oropharyngeal and retroglossal obstructions are commonly identified in up to $54 \%$ of patients ${ }^{(6)}$.

Approximately $70 \%$ of subjects have collapse at the tongue base, which contributes significantly to the obstruction during sleep ${ }^{(7)}$. A number of surgical procedures have been developed to address tongue base obstruction including tissue debulking procedures (midline glossectomy, radiofrequency ablation) and tissue repositioning (tongue suspension, genioglossal advancement, maxillomandibular advancement (MMA)), each with varying results ${ }^{(8,9)}$.

This study was aimed to assess the updated technique for endoscope-guided coblator tongue base reduction for the treatment of patients with OSA.

\section{PATIENTS AND METHODS}

This clinical trial study included a total of 12 patients with moderate and severe OSA, attending at the Department of Otorhinolaryngology, Faculty of Medicine, Zagazig University Hospital. This study was conducted between April 2018 till August 2019.

Sample size: The study included 9 males and 3 females with a mean age of 44.83 years $( \pm 6.41)$.

Assuming that mean AHI (Apnea-hypopnea Index) in preoperative cases vs postoperative cases is $45.7 \pm 25$ vs $12-8 \pm 8.5$. The sample was calculated to be 12 case by using open epi at confidence level $95 \%$ and power of test $80 \%$.

\section{Inclusion criteria:}

Patients diagnosed with moderate to severe OSA with $\mathrm{AHI} \geq 15$. Patients with lymphatic tongue base hypertrophy as diagnosed by nasoendoscopy either isolated or combined with other areas of collapse. Patients failed or not tolerated CPAP treatment. Age between 21 and 70 years. Body mass index $(\mathrm{BMI}) \leq 35$ $\left(\mathrm{kg} / \mathrm{m}^{2}\right)$. Patients who had a history of previous upper airway surgery for the treatment of OSA.

\section{Exclusion criteria:}

Patients with severe medical illness or comorbid conditions. Patients with significant craniofacial anomalies affecting airway. Unrealistic expectations. Body mass index $(\mathrm{BMI})>35\left(\mathrm{~kg} / \mathrm{m}^{2}\right)$.

All patients were subjected to full history taking and demographic data, Epworth sleepiness scale (ESS) 
questionnaire, disease duration and medication, fiberoptic examination for upper airway, polysomnography. All the studied patients underwent coblation reduction technique for the treatment of retroglossal obstruction in combination with other palatal and/or nasal surgeries in a multilevel protocol. Subjects with retroglossal obstruction already diagnosed using drug-induced sleep endoscopy and Muller's maneuver.

In this study, Coblator II Surgery System (Arthro Care ENT, Sunnyvale, CA) and an EVac 70 coblation wand (Arthro Care ENT) were used. The tongue base was observed with a 30- degree angled, rigid endoscope (4 mm).

\section{Steps of operation:}

Examination of oropharynx, nose, and nasopharynx were done. Oropharynx was examined by tongue depressor and soft palate, uvula, both tonsiles, and base of tongue seen. Freidman's maneuver used to detect the grade of obstruction. Nose and Nasopharynx was examined by Nasolaryngoscpe. Nasal septum, turbinates, post-nasopharyngeal wall were seen. Muller's maneuver used and the sites of collapse were detected. Patients were seen with FTP grade 3 and 4 included in this study and prepared for surgery.

By a wake endoscopy: Patients in sitting position and the flexible endoscopy introduced though the nostril to examine the nose, nasopharynx, and hypopharynx.

Nose: the turbinates and nasal septum were examined.

Nasopharynx: the velum, uvula, tongue base and lingual tonsils were examined. The tongue base and lingual tonsils were hypertrophied.

Hypopharynx: the epiglottis was examined and the collapse was seen at this level.

By Drug - induced sleep endoscopy (DISE): After admitted the patient to operation theatre and before began the surgery, patient placed on the theatre bed on supine position. Anaesthetic colleague started induction with propofol dose, $1 \mathrm{mg} / \mathrm{kg}$; increasing rate of $20 \mathrm{mg}$ every $2 \mathrm{~min}$. Patient underwent in sleep, at this moment, the endoscope was inserted and we examined the nasopharynx at level of velum and descended towards the tongue base and epiglottis. Upper airway collapse seen and the patient started snoring. Most of patients in this study were complaining of severe OSA and other patients were complaining of moderate OSA.

PSG system used in this study was SOMNOscreenTM plus - home and ambulatory PSG.

\section{Surgical technique:}

The procedure was performed with the patient under general anesthesia with nasotracheal intubation was introduced in the supine position with the head extended and neck sniffing position. A sheet of gauze was inserted between the lower teeth and the ventral side of the tongue to avoid a tongue laceration. Maclovr mouth gag was applied to open the mouth, and the tongue dorsum was depressed in most cases by a medium-length tongue blade to expose the base of the tongue.

A blade was used at the midline of the tongue to keep the tongue shape. We changed the blade until we got the optimal visualization of tongue base. The blade was suspended with bridge for the best field to see the tongue base (Figure 1).

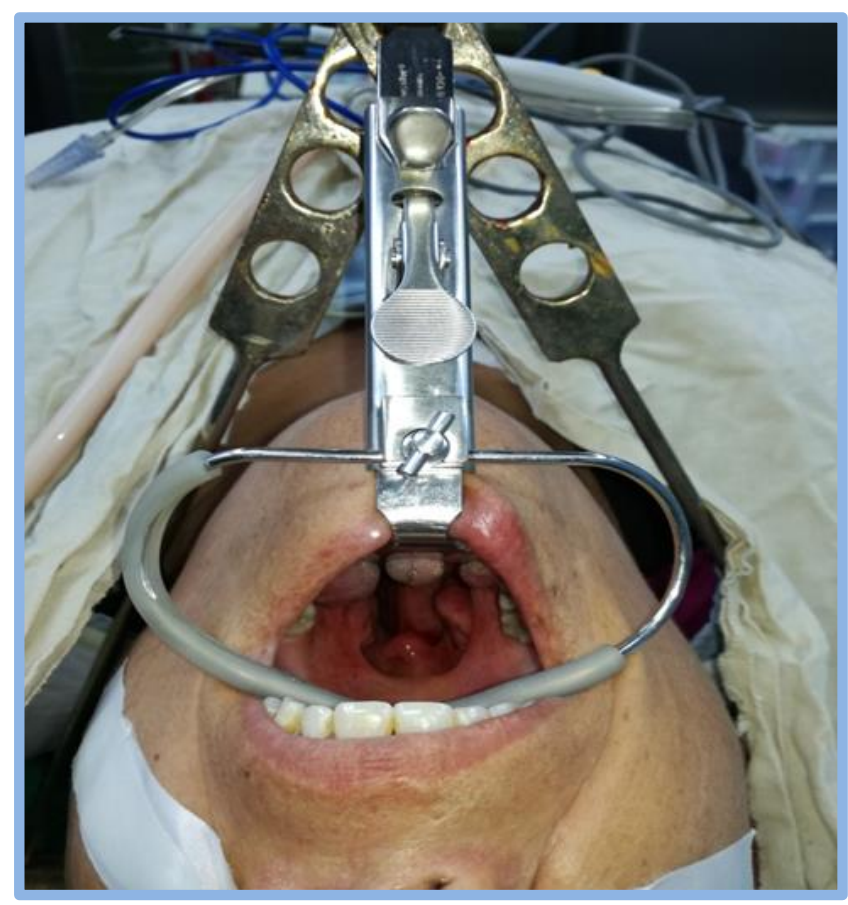

Figure (1): Mclovr mouth gag inserted and suspended by bridge.

300 endoscope connected to storz telecam SL II camera system introduced in the mouth to see the tongue base. The coblator II system prepared and connected with cold saline and suction machine. Evac70 wand was used and the shaft was bented to reach easily to the tongue base.

They applied the coblation setting of 7 and the coagulation setting of 3 in our surgeries. The tongue base was resected at posterior border from lateral side. The area from circumvallate papillae of the tongue over the midline removed by Evac70 wand and any bleeding occurred was controlled it by coagulation setting of coblator II system Figure (2). 

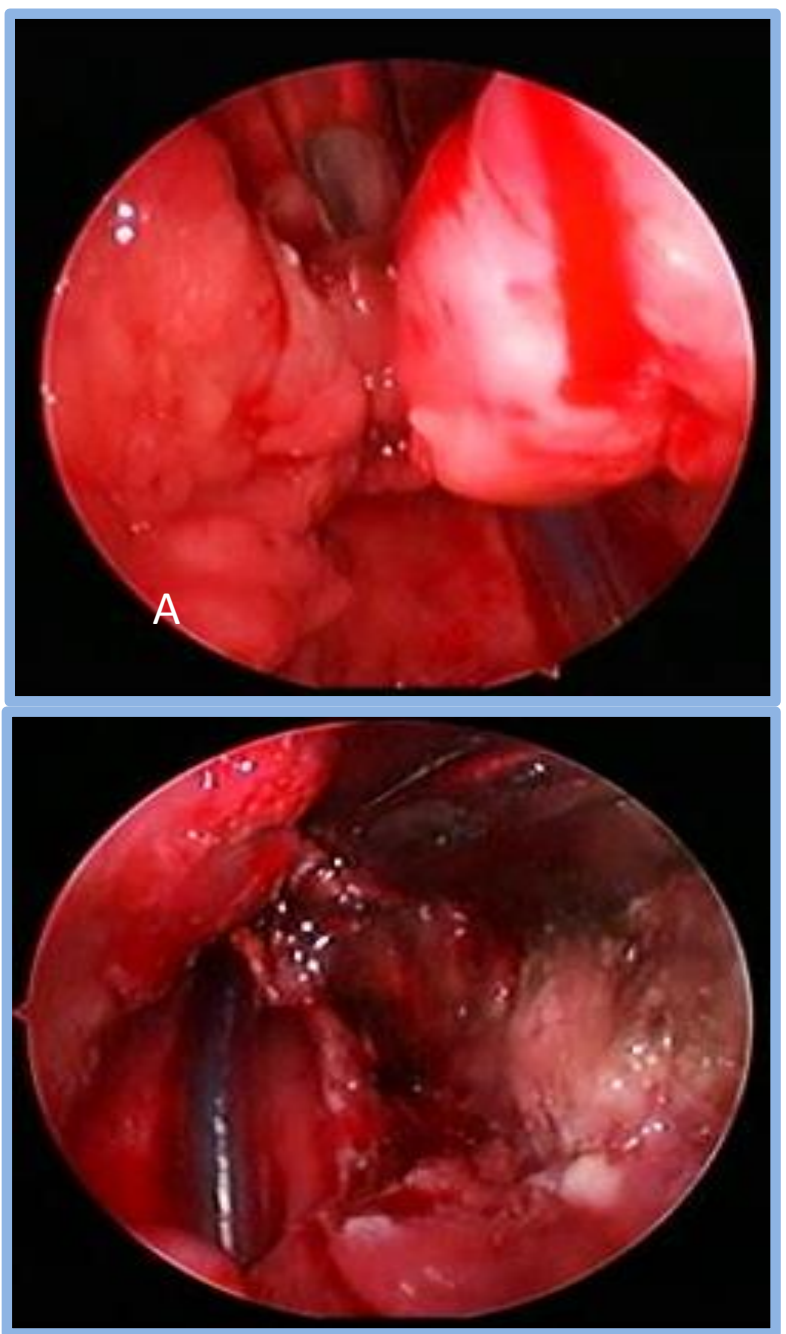

Figure (2): a -Preoperative tongue base reduction . b -Postoperative tongue base reduction by coblation.

\section{Postoperative medication:}

System antibiotics and steroid were given. All patients were given analgesics paracetamol (perfalgan) soon after operation and were allowed to eat regular foods at the first day after the operation. PPI (antireflux) was given to all patients.

After checking their health status, most of them were allowed to leave the hospital and go to their homes on the same day of the operation. Some of the cases were kept in the department and were not allowed to leave until their health status was noticed especially if tracheostomy done.

\section{Postoperative follow up:}

The first follow-up of all patients was scheduled one week after the operation. The second follow-up was two months after the date of the operation. All cases were asked to bring a post-operative sleep study to compare them with the results of the sleep study before the operation. Epworth sleepiness scale postoperation was measured for all cases and compared to the results obtained preparation.

\section{Ethical Clearance:}

Written Informed consent was taken from the patient to participate in the study. Approval for performing the study was obtained from Otorhinolaryngology Departments, Zagazig University Hospitals after taking Institutional Review Board (IRB) approval.

The work has been carried out in accordance with the code of ethics of the world medical association (Decleration of Helsinki) for studies involving humans.

\section{Statistic analysis}

Data analysis was performed using the software SPSS (Statistical Package for the Social Sciences) version 20. Quantitative variables were described using their means and standard deviations. Categorical variables were described using their absolute frequencies. Kolmogorov-Smirnov (distribution-type) and Levene (homogeneity of variances) tests were used to verify assumptions for use in parametric tests. To compare means of two groups, Mann Whitney test (for non-normally distributed data) and independent sample $t$ test (for normally distributed data) were used. Paired t test (for normally distributed data) Wilcoxon signed rank test (for not normally distributed data) were used to measure difference in the studied parameters pre and postoperatively within each group. The level statistical significance was set at 5\% (P < 0.05 ). Highly significant difference was present if $\mathrm{p} \leq$ 0.001 .

\section{RESULTS}

Male gender represents $75 \%$ of the studied patients. Age of the studied patient ranged from 38 to 54 years old with mean age 44.83 years $( \pm 6.41), 25 \%$ of the studied patients had past history of septoplasty and uvulopalatopharyngoplasty (UPPP) (Table 1). 
Table (1): Distribution of the studied patients according to demographic characteristics.

\begin{tabular}{|l|c|c|}
\hline \multicolumn{1}{|c|}{ Demographic characteristics } & $\mathbf{N = 1 2}$ & \% \\
\hline \hline Gender: & 9 & 75 \\
Male & 3 & 25 \\
Female & \multicolumn{2}{|c|}{$44.83 \pm 6.41$} \\
\hline Age (years): & \multicolumn{2}{|c|}{$38-54$} \\
Mean \pm SD & $\mathbf{N = 1 2}$ & \% \\
Range & 3 & 25 \\
\hline Past history of & 3 & 25 \\
\hline Septoplasty & 1 & 8.3 \\
\hline UPPP & \multicolumn{2}{|c|}{} \\
\hline Genioglossus advancement & \multicolumn{2}{|c|}{} \\
\hline
\end{tabular}

There was statistically significant difference between pre and postoperative Apnea hypopnea index. AHI significantly decreased from (35.3) preoperatively to (6.3) postoperatively. Median percent change in AHI is -66.49 ranged from -91.91 to -20 (Table 2).

Table (2): Comparison between pre and postoperative Apnea hypopnea index (AHI) among the studied patients.

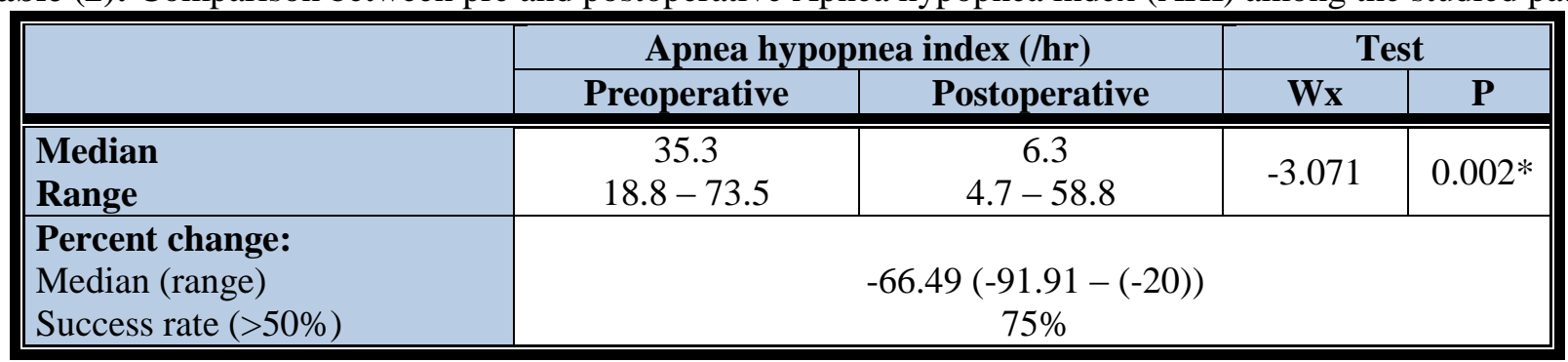

Wx Wilcoxon signed rank test $* * \mathrm{p} \leq 0.001$ is statistically highly significant

There is statistically significant difference between pre and postoperative minimum oxygen saturation. MOS significantly increased from $(76.08 \pm 18.64)$ preoperatively to $(83.17 \pm 14.5)$ postoperatively. Median percent change in MOS is 6.59 ranged from -3.45 to $78.26 \%$ (Table 3).

Table (3): Comparison between pre and postoperative minimum oxygen saturation MOS among the studied patients.

\begin{tabular}{|l|c|c|c|c|}
\hline \multirow{2}{*}{} & \multicolumn{2}{|c|}{ Minimum oxygen saturation (\%) } & \multicolumn{2}{c|}{ Test } \\
\cline { 2 - 5 } & Preoperative & Postoperative & t & P \\
\hline \hline Mean \pm SD & $76.08 \pm 18.64$ & $83.17 \pm 14.5$ \\
Range & $46-91$ & $55-97$ & -2.267 & $0.045 *$ \\
\hline $\begin{array}{l}\text { Percent change: } \\
\text { Median (range) }\end{array}$ & \multicolumn{3}{|c}{} \\
\hline
\end{tabular}

$\mathrm{t}$ paired sample t test $\quad * \mathrm{p}<0.05$ is statistically significant

There was statistically significant difference between pre and postoperative Epworth sleepiness scale. ESS significantly decreased from (10) preoperatively to (2) postoperatively. Median percent change in ESS is -79.17 ranged from -100 to $0 \%$ (Table 4).

Table (4): Comparison between pre and postoperative Epworth sleepiness scale (ESS) among the studied patients.

\begin{tabular}{|l|c|c|c|c|}
\hline \multirow{2}{*}{} & \multicolumn{2}{|c|}{ Epworth sleepiness scale } & \multicolumn{2}{c|}{ Test } \\
\cline { 2 - 5 } & Preoperative & Postoperative & Wx & P \\
\hline \hline Median & 10 & 2 & -2.821 & $0.005^{*}$ \\
Range & $8-24$ & $0-9$ & \\
\hline $\begin{array}{l}\text { Percent change: } \\
\text { Median (range) }\end{array}$ & \multicolumn{3}{|c|}{$-79.17(-100-0)$} \\
\hline
\end{tabular}

Wx: Wilcoxon signed rank test $* \mathrm{p}<0.05$ is statistically significantThere is statistically significant difference between pre and postoperative respiratory disturbance index. RDI significantly decreased from (54.3) preoperatively to (8.7) postoperatively. Median percent change in RDI is -53.72 ranged from -91.94 to $15.58 \%$

(Table 5). 
Table (5): Comparison between pre and postoperative Respiratory disturbance index (RDI) among the studied patients.

\begin{tabular}{||l|c|c|c|c|}
\hline \multirow{2}{*}{} & \multicolumn{2}{|c|}{ Respiratory disturbance index (/hr) } & \multicolumn{2}{c|}{ Test } \\
\cline { 2 - 5 } & Preoperative & Postoperative & Wx & \multirow{2}{*}{$0.005^{*}$} \\
\hline \hline Median & 54.3 & 8.7 & -2.863 & \\
Range & $18.8-73.5$ & $4.7-65$ & $-53.72(-91.94-15.58)$ \\
\hline $\begin{array}{l}\text { Percent change: } \\
\text { Median (range) }\end{array}$ & \multicolumn{3}{|c||}{} \\
\hline
\end{tabular}

Wx Wilcoxon signed rank test

$* \mathrm{p}<0.05$ is statistically significant

There was statistically significant difference between pre and postoperative ODI. ODI significantly decreased from (32.7) preoperatively to (14.8) postoperatively. Median percent change in ODI is -60.3 ranged from -87.94 to $7.03 \%$ (Table 6).

Table (6): Comparison between pre and postoperative Oxygen desaturation index (ODI) among the studied patients.

\begin{tabular}{|l|c|c|c|c|}
\hline \multirow{2}{*}{} & \multicolumn{2}{|c|}{ ODI } & \multicolumn{2}{c|}{ Test } \\
\cline { 2 - 5 } & Preoperative & Postoperative & t & P \\
\hline $\begin{array}{l}\text { Median } \\
\text { Range }\end{array}$ & 32.7 & 14.8 & -2.6 & $0.009^{*}$ \\
\hline $\begin{array}{l}\text { Percent change: } \\
\text { Median (range) }\end{array}$ & $30.3-56.9$ & $3.8-60.9$ & \multicolumn{3}{|c|}{$-60.3(-87.94-7.03)$} \\
\hline
\end{tabular}

t paired sample t test

$* \mathrm{p}<0.05$ is statistically significant

\section{DISCUSSION}

Concerning demographic characteristics of the studied patients, $75 \%$ of them were male. Mean age of them was 44.83 ( \pm 6.41$)$ ranging from 38 to 54 years.

In agreement with these results Franklin and Lindberg stated that OSA is more common in men than women ${ }^{(10)}$. Other survey again confirmed the findings of previous epidemiological studies regarding this male predominance, with this condition occurring in $70.2 \%$ of males versus $29.8 \%$ of female ${ }^{(\mathbf{1 1 1})}$.

This male predominance may be related to several factors, including hormonal effects in the muscles of the upper airways, gender-based differences in the distribution of adipose tissue, variances in pharyngeal shape, size, and collapsibility, and differences in ventilation control ${ }^{(\mathbf{1 2})}$.

The causal relationship between gender and OSA may also be confounded by exogenous exposures (i.e. occupational and environmental) and by sociobehavioral risks factors such as smoking and alcohol use. Men seek medical help late, often only when the conditions are worse, and hence tend to report more severe forms of OSA than females. Furthermore, it has been suggested that health practitioners are more inclined to suspect OSA in males than in females, leading to over-diagnosis in men ${ }^{(13)}$.

OSA related symptoms increase with age. Failure to fall asleep, frequency and duration of night awakenings and snoring are among common complaints in adults, 65 years and older ${ }^{(\mathbf{1 4})}$. The risk of OSA (AHI $\geq 10)$ for adults over 65 years is 6.6 times the risks facing those between 20 and 44 years. The prevalence of OSA $(\mathrm{AHI} \geq 10)$ in males aged 20 to 44 ,
45 to 64 and 65 and above years rose from $3.2 \%$ to $11.3 \%$ and $18.1 \%$ respectively ${ }^{(15)}$.

For both genders, the prevalence of OSA plateaued around 60 years of age, after a steady increase from younger ages. It is postulated that an increase in fat deposits in the parapharynx and comorbid conditions predispose the elderly to development of OSA. In the absence of strong evidence from longitudinal studies, it remains unclear whether OSA in the elderly is a distinct condition compared with how it presents in younger adults ${ }^{(\mathbf{1 6})}$.

Twenty five percent of the studied patients reported past history of septoplasty and UPPP. Only one patient reported history of genioglossus advancement.

Median Apnea-Hypopnea Index (AHI) significantly decreased from (35.3) preoperatively to (6.3) postoperatively. Median percent decrease in AHI is $66.49 \%$ ranged from -91.91 to -20 . The success rate was $75 \%$.

Li et al. ${ }^{(17)}$ found that AHI of their patients decreased from $48.4( \pm 16.9)$ to $44.2( \pm 19.3)$ which was by far less than improvement we found.

There is statistically significant difference between pre and postoperative minimum oxygen saturation. MOS significantly increased from (76.08 \pm 18.64) preoperatively to $(83.17 \pm 14.5)$ postoperatively. Median percent change in MOS is 5.86 ranged from -3.45 to $78.26 \%$.

Different studies agreed with ours concerning improvement in lowest oxygen saturation. The lowest O2 saturation changed significantly from $78.5 \% \pm$ $7.4 \%$ to $85.3 \% \pm 5.0 \%{ }^{(18)}$. However, minor increase in 
lowest oxygen saturation from $76.4( \pm 8.5)$ to 77.5 ( \pm 7.9$)$ was reported in the study by Li et al. ${ }^{(17)}$.

The mean minimal oxygen saturation significantly increased from $78.0 \pm 9.1$ to $83.1 \pm 6.4 \%$ $(\mathrm{P}=0.001)$ after surgery ${ }^{(\mathbf{1 9})}$.

There is statistically significant difference between pre and postoperative Epworth sleepiness scale. ESS significantly decreased from (10) preoperatively to (2) postoperatively. Median percent change in ESS is $-79.17 \%$ ranged from -100 to $0 \%$.

In contradict with our study, ESS score increased from $10.9( \pm 4.7)$ to $11.4( \pm 5)$ postoperatively (17).

There was statistically significant difference between pre and postoperative respiratory disturbance index. RDI significantly decreased from (54.3) preoperatively to (8.7) postoperatively. Median percent change in RDI is -53.72 ranged from -91.94 to $15.58 \%$.

There was statistically significant difference between pre and postoperative ODI. ODI significantly decreased from (32.7) preoperatively to (14.8) postoperatively. Median percent change in ODI is 60.3 ranged from -87.94 to $7.03 \%$.

The mean oxygen desaturation index (ODI) also significantly decreased from $31.7 \pm 20.7 / \mathrm{h}$ to $14.7 \pm$ 13.1/h $(\mathrm{P}=0.001)^{(\mathbf{1 9})}$.

Concerning length of hospital stay, 6 patients $(50 \%)$ discharged on the same day of operation. Three patients stayed for two days (25\%) and the rest 3 stayed for 10 days postoperatively. Median length of hospital stay was 1.5 days ranging from 1 to 10 days. However, all of the studied patients started normal diet on the same day.

This study had various strength points including being a prospective, randomized, clinical trial. To our knowledge, it is the first study to assess safety and efficacy of such approach in Zagazig University with no published studied on it in Egypt.

The present study had some limitations including relatively small sample size, lack of long term follow up and also lack of control group to verify the genuine role of tongue base surgery in the treatment of OSA. Lack of possibilities in terms of the lack of a coblation wand for the high price.

\section{CONCLUSION}

It could be concluded that tongue base reduction by coblation guided with endoscopy can be safe in management of obstructive sleep apnea without any dangerous complications. This approach is very safe and effective in reducing AHI, RDI, ESS and ODI. It also helps in increasing minimum oxygen saturation.

\section{RECOMMENDATION}

We recommend applying further large scale metacentric clinical controlled trial to verify our findings.

\section{REFERENCES}

1. Chen Q, Hayman L, Shmerling et al. (2011): Characteristics of chronic pain associated with sleep difficulty in older adults: the Maintenance of Balance, Independent Living, Intellect, and Zest in the Elderly (MOBILIZE) Boston study. Journal of the American Geriatrics Society, 59(8): 1385-1392.

2. Yaffe K, Laffan A, Harrison S et al. (2011): Sleep-disordered breathing, hypoxia, and risk of mild cognitive impairment and dementia in older women. Jama., 306(6): 613-619.

3. Morin CM, Vallières A, Guay $B$ et al. (2009): Cognitive behavioral therapy, singly and combined with medication, for persistent insomnia: a randomized controlled trial. JAMA., 301(19): 2005-15.

4. Eckert D, Jordan A, Merchia P et al. (2007): Central sleep apnea: pathophysiology and treatment. Chest, 131(2): 595-607.

5. Patil S, Schneider H, Schwartz A et al. (2007): Adult obstructive sleep apnea: pathophysiology and diagnosis. Chest, 132(1): 325337.

6. Lin H, Friedman M, Chang $\mathrm{H}$ et al. (2008): The efficacy of multilevel surgery of the upper airway in adults with obstructive sleep apnea/hypopnea syndrome. The Laryngoscope, 118(5): 902908.

7. Kezirian E, White D, Malhotra A et al. (2010): Interrater reliability of drug-induced sleep endoscopy. Archives of Otolaryngology-Head \& Neck Surgery, 136(4): 393-397

8. Babademez M, Ciftci B, Acar B et al. (2010): Lowtemperature bipolar radiofrequency ablation (coblation) of the tongue base for supine-position-associated obstructive sleep apnea. ORL., 72(1): 51-55.

9. Fletcher A, Choi J, Awadalla M et al. (2013): The effect of geniglossal advancement on airway flow using a computational flow dynamics model. The Laryngoscope, 123(12): 3227-3232.

10. Franklin K, Lindberg E (2015): Obstructive sleep apnea is a common disorder in the population - a review on the epidemiology of sleep apnea. Journal of Thoracic Disease, 7(8): 1311-16.

11. Wali S, Abalkhail B, Krayem A (2017): Prevalence and risk factors of obstructive sleep apnea syndrome in a Saudi Arabian population. Annals of Thoracic Medicine, 12(2): 88-96.

12. Kapsimalis F, Kryger M (2002): Gender and obstructive sleep apnea syndrome, part 2: mechanisms. Sleep, 25(5): 497504.

13. Banno K, Manfreda J, Walld $\mathrm{R}$ et al. (2006): Healthcare utilization in women with obstructive sleep apnea syndrome 2 years after diagnosis and treatment. Sleep, 29(10): 1307-1311.

14. Punjabi N (2008): The epidemiology of adult obstructive sleep apnea. Proceedings of the American Thoracic Society, 5(2): 136-143.

15. Bixler E, Vgontzas A, Ten Have T et al. (1998): Effects of age on sleep apnea in men: I. Prevalence and severity. American Journal of Respiratory and Critical Care Medicine, 157(1): 144-148.

16. Kim S, Kim B, Han J et al. (2015): Major factors affecting severity of obstructive sleep apnea. Indian Journal of Otolaryngology and Head \& Neck Surgery, 67(1): 114-118.

17. Li H, Lee L, Kezirian E (2016): Efficacy of coblation endoscopic lingual lightening in multilevel surgery for obstructive sleep apnea. JAMA Otolaryngology-Head \& Neck Surgery, 142(5): 438-443.

18. Cho H, Park D, Min H et al. (2016): Endoscope- guided coblator tongue base resection using an endoscope- holding system for obstructive sleep apnea. Head \& Neck, 38(4): 635639.

19. Wee J, Tan K, Lee W et al. (2015): Evaluation of coblation lingual tonsil removal technique for obstructive sleep apnea in Asians: preliminary results of surgical morbidity and prognosticators. European Archives of Oto-RhinoLaryngology, 272(9): 2327-2333. 